\title{
Modernidad e individuación laboral: algunas reflexiones desde la dimensión lugar de trabajo
}

\author{
Modernity and labor individuation: \\ some reflections from the workplace dimension
}

Ricardo Jorquera Gutiérrez ${ }^{1}$

Resumen

El siguiente es un artículo teórico que discute respecto al impacto de la modernidad tardía en la vida de los trabajadores en Chile, centrándose específicamente en el concepto de individuación propuesto por autores como Giddens o Beck. El análisis se hace desde la categoría "lugar de trabajo", con lo cual se observan los procesos de elección que el trabajador realiza procurando generar un curso estratégico de su trayectoria laboral. Para esto se utiliza como ejemplo demostrativo la conmutación interregional con fines laborales y el teletrabajo. Desde estos casos, se reflexiona respecto a los avances y limitaciones de los procesos de individuación de los trabajadores en Chile. Finalmente se proponen las bases de un concepto de individuación laboral.

Palabras Clave: modernidad tardía, individuación, lugar de trabajo.

\begin{abstract}
The following is a theoretical article that discusses the impact of late modernity in the life of the workers in Chile, focusing specifically on the concept of individuation proposed by authors such as Giddens or Beck. The analysis is done from the category of "work place", observing the processes of choice that the worker performs trying to generate a strategic course of his career. Interregional commutation for employment purposes and teleworking are used as demonstrative
\end{abstract}

1 Universidad Santo Tomás. Copiapó, Chile. E-mail: rjorquera@santotomas.cl 
examples. From these cases, a reflection is proposed, regarding the progress and limitations of the processes of workers' individuation in Chile. At the end, the basis for a concept of work individuation is proposed.

Keywords: late modernity, individuation, workplace.

Los cambios culturales que han afectado al mundo las últimas décadas han generado una serie de tensiones en los individuos. Buena parte de estas tensiones se relacionarían con la cada vez mayor centralidad del individuo en la generación de sus propias autobiografías e identidades personales, y en el mantenimiento de sus proyectos reflexivos de sí mismo (Giddens, 1996).

La relación entre los individuos y el ámbito del trabajo no estaría exenta de estas tensiones. Uno de los ejemplos de esto son las dificultades que los trabajadores tienen hoy en día para equilibrar su vida familiar y laboral (Jiménez \& Moyano, 2008).

En Chile, desde algunos años han aparecido reportes de investigaciones que han dado cuenta de las dificultades de los trabajadores para generar sus propias trayectorias laborales. En esta línea se destacan, por ejemplo, los trabajos de Soto (2011), quien analizó las narrativas de profesionales "de elite" de organizaciones inmersas en el mercado de trabajo flexible acerca de sus propias trayectorias laborales. También en esta dirección es necesario mencionar los aportes de Sisto (2009), quien estudió las construcciones de identidad laboral en profesionales adultos jóvenes en condiciones de vinculación laboral flexible. Por su parte, han sido importantes las contribuciones que han tratado las trayectorias laborales desde un enfoque de género (Godoy \& Mauro, 2001).

Bajo esta perspectiva de análisis, el presente trabajo pretende reflexionar respecto al verdadero poder y voluntad que los trabajadores poseen para generar sus propias vidas laborales en Chile. Específicamente se utiliza como categoría analítica el "lugar de trabajo" y su cercanía o lejanía con el hogar. El supuesto básico que sustenta la discusión desde 
esta posición, es que esta categoría debería permitir la observación de los marcos de decisión del trabajador como individuo autónomo y libre, y por tanto, debería reflejar los procesos de individuación del trabajador frente a las decisiones implicadas en elegir donde trabajar. O sea, en este caso, si es o no consciente de la elección de donde trabajar, si se siente atado o no de tomar una determinada dirección laboral, y de la vinculación de estas decisiones con la narrativa del trabajador respecto a su propia autobiografía laboral. Estas preguntas se complejizan en virtud de un escenario en el cual se evidencian la coexistencia de un mercado laboral minoritario, compuesto por personas de alta calificación y con buenas condiciones de trabajo, junto con un mercado donde se reconoce la precariedad, las bajas remuneraciones, y en donde los desarrollos de carrera en el tiempo son escasas (Soto, 2009).

Para estos efectos, a continuación se realizará una síntesis de la discusión teórica respecto a la relación entre modernidad y trabajo, para luego dar cuenta del concepto de individuación bajo este mismo prisma. Luego, se dará paso al desarrollo de la discusión de la categoría "lugar de trabajo" desde dos extremos: por un lado, se aborda la conmutación interregional, que está asociado al recorrido de largos kilómetros para llegar finalmente al lugar de trabajo. Por el otro, el "teletrabajo", que puede tener la forma de trabajo en el mismo lugar de residencia.

Finalmente, se discuten en las conclusiones algunas ideas que deberían conformar un constructo de individuación aplicado al mundo del trabajo.

\section{Modernidad y trabajo}

La modernidad es "un modo de vida u organización social que surgieron en Europa desde alrededor del siglo XVII y cuya influencia, posteriormente, lo han convertido en más o menos mundiales" (Giddens, 1994, p. 15). Esta tiene sus bases institucionales en cuatro elementos: la industrialización, la cual se refiere a "las relaciones sociales que lleva consigo el empleo generalizado de la fuerza física y la maquinaria en los procesos 
de producción” (Giddens, 1995. p. 27); el capitalismo, entendido como un "sistema de producción de mercancías que comprende tanto los mercados de productos competitivos como a la transformación en mercancía de la fuerza de trabajo" (p. 27); las instituciones de vigilancia, entendiendo vigilancia como "control supervisor de las poblaciones sometidas, tanto si este control adopta la forma de supervisión visible, como si se trata del empleo de la información para coordinar las actividades sociales" (p. 27); y los medios de coacción, los cuales están concentrados dentro de precisas fronteras territoriales (Giddens, 1994).

El curso de la modernidad no ha sido lineal ni estable, y ha tenido un dinamismo que ha generado cambios radicales en los tiempos contemporáneos. En esta lógica, Beck (2002) realiza una distinción entre lo que él denomina primera y segunda modernidad. La primera, basada en los estados-naciones, y en que las relaciones, redes sociales y comunidades se entienden esencialmente en un sentido territorial, da paso a la segunda modernidad, en donde interactúan cinco procesos interrelacionados: globalización, individualización, la revolución de los géneros, el subempleo y los riesgos globales. En estos procesos de modernización radicalizada colapsa la idea misma de controlabilidad, certidumbre y seguridad, propios de la primera modernidad. Esto tiene como consecuencia un nuevo tipo de orden global, una nueva economía, un nuevo tipo de sociedad y de vida personal.

En la modernidad encontramos separados el tiempo y el espacio, y este vaciamiento es fundamental para entender el desenclave de las instituciones sociales, nombre que da a entender "la extracción de las relaciones sociales de sus circunstancias locales y su rearticulación en regiones espacio-temporales indefinidas" (Giddens, 1995, p. 30). Sin lugar a dudas, uno de los mecanismos que han favorecido los cambios globales que viven las personas hoy en día, y que ha conllevado estas nuevas maneras de interacción social, es la propagación de las redes informáticas, las cuales han permitido una comunicación cada vez más apresurada, en la cual las personas pueden estar conectadas no necesitando depender de un mismo espacio y tiempo. 
Manuel Castells (1999) ha descrito cómo los cambios en los planos de las tecnologías de la información sientan las bases para una nueva economía de escala mundial. Esta nueva economía se caracteriza por tres rasgos: en primer lugar, su carácter informacional, lo cual hace referencia a que las unidades que componen esta nueva economía (empresas, naciones, regiones) y su productividad y competitividad, dependen directamente del poder que tengan para generar, procesar y aplicar la información basada en el conocimiento. En segundo lugar, su aspecto global, pues la producción, el consumo y su circulación, se encuentran dentro de una organización a escala global. Y en tercer lugar, su conexión en red, la que permite las interacciones globales en donde se va a desarrollar la productividad y la competencia. La economía global se caracteriza por tener la capacidad institucional, organizativa y tecnológica para funcionar como una unidad a tiempo real o tiempo establecido a escala planetaria. En este escenario, se hace crucial el cerebro humano en el proceso de trabajo. Tienden a disminuir las tareas repetitivas, gracias a la automatización de los procesos, y se revalorizan el trabajo analítico, el de decisión y programación. Otro aspecto importante para el mundo laboral, es el crecimiento del trabajo flexible. Este implica cuatro aspectos: primero, la flexibilización de la jornada laboral; en segundo lugar, el empleo se centra en la tarea en sí, y no en un compromiso con la empresa; en tercer lugar, cada vez más aumenta el trabajo fuera de la sede de la empresa; y en cuarto lugar, se comienza a observar una disminución de la relación tradicional centrado en la fidelidad entre empleado y empresa.

En general, existe un cambio mundializado que marca una diferencia notable en las formas de las estructuras organizativas y culturales en la empresa moderna. Uno de los aspectos más notable, que configura la forma de relacionarse de los individuos con las organizaciones, es que en las últimas décadas se hace evidente un debilitamiento de la vinculación identitaria de los trabajadores con las instituciones. Hoy en día las personas no constituyen su identidad de manera preferente en relación a las instituciones sociales como la religión, el partido político o la nación. Asimismo, tampoco los trabajadores estructuran su identidad en referencia a las or- 
ganizaciones laborales. En esta línea Richard Sennett (2007) plantea que la nueva estructura de la organización capitalista implica una disminución importante del capital social manifestado en la relación de los trabajadores con las empresas. Este autor observa tres grandes déficit producidos por el cambio estructural: la baja lealtad institucional, la disminución de la confianza informal entre los trabajadores y el debilitamiento del conocimiento institucional. Hoy día los trabajadores tienden a no generar redes de lealtades entre empresas y trabajadores. Estos déficits tienen como resultado que los equipos de trabajo se transforman en redes que se pueden deshacer fácilmente.

Junto con los efectos del nuevo capitalismo sobre los procesos de identidad en el individuo, el neocapitalismo afecta también la acelerada obsolescencia de los conocimientos y las destrezas adquiridas, producida por el frenético cambio en las innovaciones productivas y tecnológicas. Hoy en día, cualquier profesional medio se ve en la obligación de reciclarse dos o tres veces a lo largo de su vida laboral, la cual tiende a prolongarse cada vez más (Sennett, 2007).

Lo anterior muestra una profunda contradicción, pues mientras la flexibilización y el dinamismo de la producción globalizada abren una serie de posibilidades en el mundo del trabajo en algunos sectores (por ejemplo, para los analistas simbólicos), asociadas con conceptos como autonomía y creatividad, la reorganización del trabajo abre vulnerabilidades y precariedades, principalmente vinculadas a la reducción de puestos de trabajo en sectores tradicionales, dado que el nuevo sector no es intensivo en mano de obra sino en tecnología (Rifkin, 1999, citado en Hopenhayn, 2004). Sin lugar a dudas, el temor del desempleo genera una serie de problemáticas en la subjetividad relacionadas con la incertidumbre y el reconocimiento de la vulnerabilidad personal.

Por tanto, el debilitamiento del capital social en las organizaciones y la ansiedad por no quedar obsoleto o derechamente marginado del mercado del trabajo, producen en los individuos una serie de tensiones que hacen cada vez más áridos los desarrollos de carrera de los trabajadores. Como se verá en el apartado siguiente, en la modernidad 
reflexiva el individuo debe ser más activo en el desarrollo de sus biografías personales.

\section{Individuación}

Desde el punto de vista de la vida de las personas, la modernidad ha alterado de manera radical la naturaleza de la vida social cotidiana, afectando los aspectos más personales de nuestra experiencia (Giddens, 1995). En palabras de Touraine (1998):

el mundo moderno está cada vez más penetrado por un sujeto que es libertad, es decir, que postula como principio del bien el control que el individuo ejerce sobre sus actos y su situación y que le permite concebir y sentir su conducta como componente de su historia personal de vida, concebirse él mismo como actor. El sujeto es la voluntad de un individuo de obrar y de ser reconocido como actor (p. 207).

Hoy día los individuos no se constituyen en relación a las instituciones sociales, sino, generan sus trayectorias personales en referencia a sus propios procesos de reflexividad. Es más, incluso en la relación con los otros, espacio que constituía la unidad básica en donde clásicamente se han establecido las identidades personales, lo que prima es la facilidad con la cual se puede deshacer la red de relaciones. Como plantea Bauman (2008) en la "Modernidad líquida", en los tiempos actuales "el verdadero énfasis se pone en la facilidad para desmantelar los vínculos, en vínculos que son tan fáciles de romper como de forjar" (p. 190). Hoy los individuos tienen muchas más oportunidades para configurar su propia vida, en las cuales pueden constituirse activamente y desarrollar su propia identidad. Esto se da gracias a que los marcos identitarios tradicionales se están disolviendo y emergen nuevas pautas de sentido. La globalización obliga a los individuos a vivir de una forma abierta y reflexiva, dado que deben responder al contexto general en el cual viven (Giddens, 2004).

En contextos de modernidad reflexiva cobra sentido el concepto de individuación, el cual revela "los contenidos en los cuales el sujeto se re- 
laciona con el medio de manera específica y autorregulada, interactuando con las tramas culturales de forma proactiva y sin una sujeción que implique inmovilismo frente a las dinámicas de la cultura" (González, Kreither, Lizana, Rodríguez \& Zavala, 2009, p. 5). La individuación implica reconocer al individuo como "actor, diseñador, malabarista y director de escena de su propia biografía, identidad, redes sociales, compromisos y convicciones" (Beck, 2001, p. 29), y encierra que el propio individuo busque sus nuevas certezas, dada la caída de las certezas de la sociedad tradicional. Además de esto, significa para el individuo la generación de nuevas interdependencias, incluso de tipo global. La individualización en Beck (2001) significa una compulsión a fabricar, autodiseñar y auto-escenificar no solo la propia biografía, sino también sus compromisos y redes de relaciones a medida que cambian las preferencias y fases de la vida. La individuación significa una biografía reflexiva (Giddens, 1994).

Dado que la individuación implica la definición personal de las biografías del yo, se encuentra relacionada con conceptos como los de estilo de vida, planificación estratégica de la vida, y de proyecto reflejo del yo.

El individuo, inserto en las condiciones de la modernidad, está enfrentado a una compleja diversidad de elecciones y, al carecer de carácter funcional, ofrece al mismo tiempo poca ayuda en cuanto a qué opción se habrá de escoger. De esto se derivan varias consecuencias, una de ellas es la primacía de un estilo de vida, considerado este como un "conjunto de prácticas más o menos integrado que un individuo adopta no solo porque satisfacen necesidades utilitarias, sino porque dan forma material a una crónica concreta de la identidad del yo" (Giddens, 1995, p. 106). Tal selección o creación de un estilo de vida está influido por presiones de grupo y por la visibilidad de los modelos de rol, así como por las circunstancias socioeconómicas.

Frente a este mundo de opciones alternativas, la planificación estratégica de vida adquiere una especial importancia, debido a que esta es un medio de preparar una línea de acción futura activada en función de la biografía del yo. La planificación de la vida supone un medio específico de organizar el tiempo, pues la construcción refleja de la identidad del yo depende tanto de la preparación para el futuro como de la interpretación 
del pasado, aunque en este proceso es siempre importante la reelaboración de los sucesos del pasado (Giddens, 1995).

Elemento fundamental del proyecto reflejo del yo en tiempos de modernidad es el cuerpo y la apariencia. Este cuerpo es "un sistema de acción, un modo de práctica, y su especial implicación en las interacciones de la vida cotidiana es parte esencial del mantenimiento de un sentido coherente de la identidad del yo" (Giddens, 1995, p. 128). Somos responsables del diseño de nuestros propios cuerpos y, en cierto sentido, nos vemos forzados a serlo cuanto más postradicionales sean los ámbitos sociales en que nos movamos.

En general, la vida en la modernidad es constitutivamente incierta, dificultad que se acentúa por la importancia de elecciones de estilo de vida. De esta manera la identidad del yo se ha de crear y reordenar sobre el trasfondo de las experiencias cambiantes de la vida diaria y de las tendencias fragmentarias de las instituciones modernas (Giddens, 1995). Frente a un panorama como este, vivir en el mundo de la modernidad implica diversas tensiones y dificultades específicas en el yo, teniendo que resolver distintos dilemas con tal de preservar una crónica coherente en su identidad. Por un lado la modernidad fragmenta, pero también une. Tal fragmentación está estimulada por la diversidad de las circunstancias de interacción, en donde el individuo debe acomodar la "presentación de su yo" de acuerdo a los requerimientos de cada situación concreta. Sin embargo, una persona puede aprovechar la diversidad para crear una identidad propia que incorpore favorablemente elementos de diferentes ámbitos en una crónica yoica integrada.

Aunque la modernidad da apertura al proyecto reflejo del yo, lo hace en condiciones fuertemente influenciadas por el capitalismo mercantilista.

Los mercados estimularon desde el primer momento el individualismo, haciendo hincapié en los derechos y responsabilidades individuales, pero al principio este fenómeno afectó principalmente a la libertad de contrato y a la movilidad intrínseca que caracteriza al empleo capitalista. Luego, sin embargo, el individualismo se extendió a la esfera del consumo, pues para la continuidad del sistema es fundamental determinar los deseos del 
individuo. La libertad de elección individual, dirigida por el mercado, se convierte en un marco envolvente de expresión individual del yo (Giddens, 1995, p. 250).

Individuación y trabajo en Chile: Reflexión desde la categoría el lugar de trabajo

El tema de la distinción entre lugar de trabajo y residencia es típicamente moderno. Se hace masivo con los procesos de industrialización en el siglo XVIII. Antes de esto, mayoritariamente existía una comunión entre residencia y trabajo. El orfebre, el campesino, el herrero, y en general buena parte de los artesanos residían en el mismo lugar en el cual trabajaban. Con el surgimiento de la industrialización, el trabajador se vio obligado a comenzar a trasladarse hacia su lugar de trabajo, con lo cual se hizo patente la separación entre hogar/trabajo.

Para problematizar reflexivamente cómo se desarrollan los procesos de individuación en el ámbito del trabajo en Chile se describirán dos ámbitos laborales en los cuales se evidenciarían algunas de sus características: la conmutación interregional y el teletrabajo. Esto bajo el supuesto que la elección de viajar largas distancias, o de reducir al máximo el tramo recorrido entre el trabajo y el lugar de residencia, implica una decisión consciente en la cual se expresaría el proyecto autobiográfico en relación al trabajo. La posición que se defiende es que ambas formas de trabajo son dos caras de la misma moneda; dos formas derivadas de las características del trabajo en la modernidad tardía, en la cual finalmente hay un des-anclaje entre lugar de trabajo y residencia cada vez más radicalizado. En el caso de la conmutación este des-anclaje es claramente espacial y físico, y en el caso del teletrabajo es un distanciamiento simbólico, que se basa en la distinción hogar/trabajo. Lo que queda para el análisis posterior son los efectos bidireccionales que tiene el fenómeno de distanciamiento entre lugar de trabajo y residencia, y los procesos de individuación de los trabajadores.

Para observar estas dos formas de trabajo con los ojos de los códigos de la modernidad reflexiva es prudente realizar la siguiente pregunta: 
la conmutación interregional y el teletrabajo, ¿elección consciente y libre, o conformismo y resignación con la realidad que se vive? Esta pregunta cobra sentido frente a un panorama cultural en el cual predomina como centralidad el individuo, el cual es el motor y objetivo de los cambios a nivel de las propias vidas.

La contestación positiva a este asunto permitiría dilucidar que efectivamente en Chile estamos en presencia de un trabajador moderno, actor y conductor de su propia historia. Este sería un individuo que es capaz de generar un trabajo autoprogramable (Castells, 2001). Sin embargo, es posible suponer que no todos los trabajadores lograrían este nivel de libertad y autonomía para desarrollar su trabajo y generar sus propias trayectorias laborales. Castells (2001) plantea que el trabajo autoprogramable requiere un alto nivel educativo y una alta capacidad de tomar iniciativas; pero además, que el trabajador sea capaz de expandir y modificar sus conocimientos y habilidades a lo largo de toda su vida activa. Por ello, una de las características más importantes de este nuevo trabajador es que sea capaz de aprender a aprender, ya que la mayor parte de las informaciones específicas quedarán obsoletas en pocos años dado el dinamismo de la nueva economía. La otra característica consiste en estar capacitado para transformar la información obtenida durante el proceso de aprendizaje en conocimiento específico.

Junto con los cambios a nivel de los trabajadores, el nuevo tipo de trabajo autoprogramable requiere ciertas condiciones institucionales para que pueda llevarse a cabo. De manera específica, se requiere un entorno empresarial flexible, esto es, una jerarquía plana, un sistema de trabajo en equipo y una interacción abierta y fácil entre trabajadores y gestores, entre departamentos y entre los diversos niveles de la empresa (Castells, 2001).

\section{Conmutación Interregional}

En primer lugar abordaremos la conmutación interregional con fines laborales. La conmutación es entendida como el viaje de ida y vuelta que el trabajador realiza entre su residencia y su lugar de trabajo. Este fenómeno empezó a extenderse con la industrialización y fue favorecida por la 
reducción progresiva de los costos de transportes, la concentración urbana y el aumento de la mano de obra asalariada. Al principio la conmutación fue caminando, y luego, los medios de transporte se extendieron al ferrocarril y más tarde al automóvil y el avión. En los últimos años este fenómeno se ha mundializado y su forma se ha diversificado en cuanto a medios de transportes, tiempos y distancias (Aroca \& Atienza, 2008).

En Chile se ha observado un incremento de la conmutación interregional de larga distancia. Aroca \& Atienza (2007) han constatado, usando datos del censo 2002, que la migración laboral interregional llega a un $1,31 \%$ de la población activa, mientras que la conmutación entre regiones alcanza el 2,54\% del mismo grupo. La Región Metropolitana es la que registra una mayor actividad de conmutación, seguida por la región de Antofagasta, esta última asociada a la industria minera. En la segunda región, casi el 10\% de la fuerza laboral conmuta desde otras regiones. Aroca \& Atienza (2007) han diferenciado las regiones entre aquellas que son atractivas para trabajar y no para residir, y viceversa. Las regiones IV, $\mathrm{V}$ y VI muestran una migración neta positiva y una conmutación regional negativa, esto quiere decir que estas regiones son elegidas para vivir, pero las personas tienden a preferir trabajar en otras regiones. A diferencia de estas, las regiones I, III y XII poseen una migración neta negativa y una conmutación positiva, o sea, estas regiones no son atractivas para vivir, pero sí lo son para trabajar. Por su parte, las regiones II, XI y X poseen una migración y conmutación positiva, siendo atractivas tanto para vivir como para trabajar. Esto muestra que existe conmutación positiva en las regiones del norte, sur y metropolitana, mientras las regiones del centro tienen conmutación negativa.

En Chile, el tema de la conmutación ha sido estudiado principalmente referido a los efectos económicos que implica para las regiones. Aroca \& Atienza (2008) observaron el impacto económico de la conmutación, especialmente en la región de Antofagasta, la cual implica efectos directos e indirectos, como pueden ser los referidos a obtener un sueldo en una región y utilizarlo en un consumo que se realiza en otra. Pero también ha sido observada la implicancia de la conmutación sobre los incentivos para 
la acumulación de capital social en las regiones que reciben a los trabajadores que conmutan, debido a la movilidad, a la distancia que los separa de sus redes familiares y a la falta de propiedad de una vivienda en las regiones destino, todos ellos factores relacionados negativamente con la formación de capital social (Glaeser, Laibson \& Sacerdote, 2000, citado en Aroca \& Atienza, 2007).

Una de las principales razones de la conmutación interregional en Chile está asociada al desplazamiento de trabajadores hacia el sector minero, cuyas faenas se encuentran habitualmente alejadas de centros urbanos, en sectores cordilleranos. Al respecto es necesario mencionar que el crecimiento sostenido de la minería ha implicado la expansión de las faenas a estas zonas, concentrándose el $80 \%$ del total de las faenas de las empresas mineras en ubicaciones sobre los 3.000 metros sobre el nivel del mar. Estas condiciones las obliga a operar con jornadas excepcionales de trabajo, las cuales deben ser autorizadas por la Dirección del Trabajo. Estas jornadas excepcionales consisten en ciclos de días de trabajo y descanso cuya distribución y proporción es diferente a la jornada ordinaria: por ejemplo siete días de trabajo por siete días de descanso $(7 \times 7)$; cuatro días de trabajo por cuatro días de descanso (4x4), entre otras. Según un estudio realizado por Carrasco $\&$ Vega (2011), un porcentaje importante de una muestra de trabajadores de la gran minería $(79,8 \%)$ evaluaron las jornadas excepcionales como un buen sistema de organización de las labores, que les permite concentrar los días de trabajo en un tiempo determinado y luego contar con varios días continuos de descanso, favoreciéndoles el poder compartir con la familia y disponer de tiempos personales. En este sentido, si bien reconocen que este es un sistema bastante sacrificado, valoran los días de descanso y el salario como elementos compensatorios. Por su parte, los detractores del sistema de jornadas excepcionales, evalúan las jornadas de trabajo como muy extensas y agotadoras, viendo afectadas su vida familiar y social.

Esta última visión fue observada en una muestra de mineros colombianos (Correales \& Gómez, 2011), en donde se encontró un nivel bajo de bienestar laboral, pero sobre todo un nivel muy bajo en el bienestar de pareja. Las autoras afirman que el trabajo por turnos provoca una altera- 
ción en el ámbito familiar y social, pudiendo ser una fuente importante de conflictos tales como: problemas en la relación de pareja, escaso contacto con los hijos, incompatibilidad de horarios con los amigos o problemas para disfrutar del tiempo libre, entre otros.

En esta línea, Baez \& Galdames (2005) compararon a trabajadores con y sin jornada excepcional de trabajo y observaron que los con jornada excepcional presentan mayor conflicto de rol, por lo cual estimaron que es posible pensar que estos perciben de forma más negativa la compatibilidad y ajuste en los papeles de la vida laboral y familiar. Asimismo, observaron que los trabajadores de jornada excepcional poseían, en la muestra estudiada, una menor implicancia laboral, un mayor bienestar psicológico y una mayor satisfacción laboral.

Carrasco \& Vega (2011) comentan que el disfrute por el trabajo, su adhesión a él, así como el interés por mantenerse en esta actividad, constituyen un importante factor protector de la salud mental de los trabajadores y sugiere que contribuyen a una mayor adaptabilidad al particular sistema de trabajo. Estas autoras infieren que, en general, los mineros proyectan disfrutar la vida a más largo plazo, cuando estén jubilados. En este sentido, postergan su bienestar y planes de descanso y disfrute de la vida social y familiar para el futuro.

En el caso de la conmutación interregional se evidenciaría como un acto que reflejaría los procesos de individuación laboral individual, en el sentido que implica una acción de elección en donde se escinde el plano familiar y el trabajo, en la deliberación de donde trabajar. Existe una decisión de ocuparse en alguna ciudad o zona distinta a la de residencia, con la creencia de conseguir un nivel de calidad de vida familiar que no necesariamente se conseguiría en la ciudad de trabajo. En este escenario, es posible hipotetizar que los trabajadores que se encuentran en cargos de mayor calificación y en condiciones contractuales más seguras, y tienen una mayor tranquilidad para decidir respecto a las propias trayectorias laborales. Trabajadores en condiciones de mayor precariedad contractual no tendrían la misma libertad para elegir y movilizarse libremente por el mercado laboral. Por otro lado, la alta demanda y baja oferta de profesiones 
con alta calificación, como puede ser la de un ingeniero, favorece también la flexibilidad para buscar nuevas alternativas económicas y proyecciones profesionales. En el caso de trabajadores de menor calificación profesional (técnicos de nivel medio o sin estudios especializados), la mayor libertad de elección la conseguirían mediante la especialización adquirida por experiencia y por su focalización en algún oficio escaso y altamente demandado por la industria (por ejemplo, un operador de jumbo, maquinaria de perforación ocupada para realizar túneles y obras subterráneas), la cual le facilita un mayor atractivo en el mercado y una mayor facilidad para movilizarse en esta industria.

Claramente, existe entre las personas que participan en esta industria una disposición a la conmutación, lo que sin lugar a dudas compromete de manera global a todo el sistema familiar. Por lo general, la familia permanece en la ciudad de residencia, sin la presencia del jefe de hogar por varios días, debiendo con este sistema acomodar las funciones parentales y maritales de acuerdo a los turnos laborales.

Desde un punto de vista contractual, la flexibilidad estaría mediada por aspectos vinculares clásicos, en este caso por una relación laboral de dependencia, tal cual es definida por el Código del Trabajo. En la conmutación existen contratos formales de trabajo que conectan al trabajador en una relación laboral de dependencia formal con la empresa, que limitan su flexibilidad para movilizar sus recursos y los modos de trabajo.

\section{Teletrabajo}

El otro extremo de tipos de trabajo, según la distancia que el trabajador mantiene entre su residencia y su lugar de trabajo, es el trabajo a domicilio, en donde la distancia de conmutación es cercana o igual a cero.

El trabajo a domicilio es una modalidad antigua de empleo caracterizada porque una parte del proceso productivo se elabora fuera del establecimiento de trabajo, en el domicilio del trabajador, a quien la empresa encarga la elaboración de determinados bienes o servicios o de partes de estos. Este trabajo no es comparable al trabajo que los artesanos realizaban en su 
propio hogar en la era pre-industrial, pues implica un menor nivel de autonomía y una clara subordinación. Una de las ventajas que el trabajo a domicilio posee es que emplea más fácilmente tiempo fuera de la jornada ordinaria de trabajo, lo cual permite agregar valor al proceso productivo. El trabajo a domicilio permite una importante prolongación de las jornadas de trabajo, que en el espacio formal de la empresa no es posible lograr (Henríquez, 2005).

Un tipo específico de trabajo a domicilio es el teletrabajo. Según Gareca, Verdugo, Briones y Vera (2007), en la literatura aún no se ha generado consenso para definir el teletrabajo, sin embargo convergen las ideas de diversos autores y organismos internacionales en tres características:

- Existe una relación contractual entre el empleador y el teletrabajador.

- El trabajo se desempeña en un lugar distinto a la oficina central o primaria del empleador.

- Las tecnologías de información y comunicación (TIC) son el medio indispensable para su ejecución.

Respecto a este tipo de trabajo, el artículo 22 del Código del Trabajo, en su inciso cuarto plantea su liberación de los límites de la jornada laboral, dejando vislumbrar las mismas características señaladas anteriormente: "quedan excluidos de la limitación de jornada, los trabajadores contratados para que presten sus servicios preferentemente fuera del lugar o sitio de funcionamiento de la empresa, mediante la utilización de medios informáticos o de telecomunicaciones" (Gobierno de Chile, 2003).

Cárdenas (2005) aprecia que el teletrabajo no puede ser descrito, analizado y comprendido adecuadamente desde la dicotomía "dependiente-independiente", dado que a pesar que el teletrabajo no se desarrolla en el marco de un contrato laboral, las relaciones que se establecen entre el teletrabajador y la empresa son, por lo menos en parte, de carácter dependiente. Principalmente a partir de lo denominado dependencia económica, donde uno de los elementos constitutivos es la ejecución de un trabajo en forma regular y estable (por lo menos) para una empresa, a cambio de una remuneración. Esta regularidad y su adaptación de la empresa reforzarían los lazos de dependencia entre trabajador y empleador. Se observa 
sin embargo, una tendencia a la horizontalidad de las relaciones de trabajo, lo cual favorece la autonomía del trabajador para tomar algunas decisiones respecto de este. A pesar de esto, según lo expresado por Selamé (2005), y pese a la independencia del trabajador, en el teletrabajo se estaría bajo la presencia de condiciones de subordinación económica y jurídica, pues se darían total o parcialmente la mayoría de las siguientes características: a) la prestación del servicio tiene lugar en el marco de una organización empresarial; b) esta debe realizarse conforme a las órdenes e instrucciones del dador del trabajo; c) la misma prestación del servicio implica para el prestador tener que encontrarse total o parcialmente a disposición del dador del trabajo; d) la prestación del servicio se da con cierta permanencia en el tiempo; e) el prestador del servicio debe cumplir horario; f) la empresa dadora del trabajo proporciona al trabajador documentación que la identifica.

Sin lugar a dudas en esto es posible compartir las impresiones de Henríquez (2005), quien plantea que el diagnóstico sobre el teletrabajo está construyéndose y no ha alcanzado aún precisión suficiente para definir el tratamiento jurídico adecuado. Esta autora nos señala la necesidad de lograr mayor evidencia empírica para establecer la naturaleza de esta relación laboral, pero también para mostrar cómo se va conformando la identidad laboral de este nuevo tipo de trabajadores.

Es posible reconocer, en el caso del teletrabajo, condiciones que favorecen un tipo de funcionamiento laboral con mayores niveles de flexibilidad. Sin embargo, es distinguible que estos son variables de acuerdo a las capacidades del trabajador. Es posible evidenciar que aumenta el nivel de control en la medida en que disminuye la calificación laboral del trabajador. Por otro lado, a medida que el trabajador tiene un mayor nivel educativo crece la confianza en sus calificaciones profesionales y existe una mayor horizontalidad y un menor control directo sobre sus acciones; logra mayor autonomía y mas discrecionalidad sobre la manera como ejecutará sus labores. Esto quiere decir que la libertad del trabajador está mediada por sus competencias demostrables a través de sus estudios formales.

Un segundo punto a discutir es el elemento vincular con las organizaciones laborales. En el caso del teletrabajo, si bien puede existir o no 
una relación de dependencia laboral, pues podría estar relacionada con una modalidad de trabajo freelance y/o a honorarios, existiría de igual forma una relación de subordinación jurídico/económica, tal cual como lo señala Cárdenas (2005). Podría suceder que no exista dependencia física, y que el trabajador tenga una mayor libertad de decisión respecto a la forma como administra sus tiempos, o como aborda sus objetivos, sin embargo, la regularidad del trabajo y la recepción de una remuneración sistemática por parte de la empresa marcan la dependencia.

\section{Conclusiones}

Es posible concluir que en el caso de estas realidades laborales, conmutación interregional y teletrabajo, existe un factor en común que facilitaría los procesos de individuación de los trabajadores, el cual está referido al nivel educativo y a la calificación laboral. Es posible apreciar que el trabajador que posee una mayor formación especializada deseable por el mercado, tenderá a tener mayores posibilidades de generar su propia autobiografía laboral, por tanto, de generar procesos de individuación respecto a su propia historia y trayectoria laboral. En cambio, el trabajador con menor calificación y experiencia verá disminuidas sus posibilidades para desarrollar procesos de elección autónomos de carreras laborales. Esto es problemático al pensar que la proporción de trabajadores con altos niveles de calificación, versus trabajadores con menor nivel educativo y baja capacitación técnica no es equilibrada, y tiende a ser altamente mayor la fracción de trabajadores que participan de un mercado precario que favorece las vulnerabilidades sociales y las tensiones psicológicas. Con esto, se perpetúa que el buen trabajador, en estos ámbitos, será el que logre mayores niveles de desapego, y que por tanto, pueda tomar distancia frente a las relaciones con el fin de aprovechar las oportunidades del entorno (Soto, Espinoza \& Gómez, 2008).

Estas formas de trabajo, las cuales circunscriben de manera particular la relación con la empresa, permiten observar que en Chile, los trabajadores y las empresas no son ni tan modernos, ni tan tradicionales. Lo que se aprecia es un camino evolutivo que vislumbra una mayor centralidad en el individuo, tanto en los mecanismos institucionalizados 
en el mercado, como en la propia conciencia de los sujetos. Una tendencia a rasgar ataduras con las clásicas dependencias laborales. Sin embargo, aun hay espacios institucionales en estas nuevas formas de trabajo, y estructuras psicológicas en los trabajadores, que favorecen la ligazón con la empresa de acuerdo a los vínculos clásicamente definidos como dependencia laboral, y/o como dependencia con el lugar. Algunos sujetos claramente siguen añorando nostálgicamente la estabilidad y la proyección de largo plazo (Soto, 2011).

En este sentido, cobra valor la idea de Perilleux (2008), quien sostiene que el individuo debe negociarse en el emprendimiento de sí, "despojándose de los colectivos estables y de todo vínculo duradero, corriendo el riesgo de convertirse en un sujeto flotante, que navegaría sobre la existencia creyendo poder hacer tabula rasa de su pasado, siempre preso entre la euforia del posible y la depresión del ideal" (p. 152).

Todo esto abre la necesidad de discutir respecto a la especificidad de un concepto de individuación aplicado al mundo del trabajo; un concepto que debería hacer referencia a cómo el individuo construye su trayectoria y biografía laboral, pero además, a la manera de percibirse como constructor del proyecto estratégico laboral de sí mismo. Este constructo debería facilitar la descripción de cómo el sujeto toma conciencia de su poder como edificador de su propia trayectoria laboral. Pero también, debería permitir el trabajo comparativo de diversos grupos de trabajadores de distintas industrias, género, y posiciones laborales.

Un concepto de individuación laboral (o sentimiento de individuación laboral) debería considerar a lo menos cuatro aspectos:

- Conciencia del proyecto de vida laboral personal. Implica la visualización de una estrategia personal dirigida a la colonización del futuro laboral.

- Elección de estilo de vida laboral. Referido al sentimiento de que es posible generar y/o acomodarse a un determinado estilo de vida laboral.

- Reflexividad del yo. Entendida como la disposición a revisar de manera permanente el curso del proyecto de vida laboral, 
con tal de mantener equilibradas las demandas del entorno y las necesidades personales.

- Autobiografía laboral. Conciencia de la historia laboral, y disposición a utilizarla para favorecer la reflexividad del proyecto reflejo del yo en el plano laboral.

Con esto se desea sugerir que es posible entender los procesos que viven las personas en el nuevo contexto organizacional, utilizando el constructo de individuación laboral. Con este concepto se desea generar una herramienta teórica útil para evaluar el sentimiento del individuo respecto al control y potestad que posee sobre su vida laboral. A su vez, de manera agregada, permitiría conocer la forma como se extiende la modernidad sobre el mundo del trabajo en Chile.

A modo de cierre se desea expresar dos nuevas preguntas para abrir nuevas discusiones: ¿Cómo se hace cargo la empresa de los procesos de individuación del trabajador, el cual es responsable de su trayectoria laboral, pero cuya libertad implica también tensiones en su yo? ¿Cómo se conectaría la individuación del trabajador con las tensiones de la vida familiar y de la generación de proyectos conjuntos con el resto de los miembros de la familia?

\section{Referencias}

Aroca, P. \& Atienza, M. (2007). Impacto sobre el crecimiento regional de la migración y conmutación interregional en Chile. Taller Nacional sobre Migración interna y desarrollo en Chile: diagnóstico, perspectivas y políticas. Santiago: Cepal-Celade.

Aroca, P. \& Atienza, M. (2008). La conmutación regional en Chile y su impacto en la Región de Antofagasta. Revista EURE, 34(102), 97-120.

Baez, X. \& Galdames, C. (2005). Conflicto de rol familia-trabajo desde la perspectiva de los tipos de jornada de trabajo. Revista de Psicología, 14(1), 113-123.

Bauman, Z. (2008). La sociedad sitiada. Buenos Aires: Fondo de Cultura Económica.

Beck, U. (2001). La reinvención de la política: Hacia una teoría de la modernización reflexiva. En U. Beck, A. Giddens, \& S. Lash. La 
modernización reflexiva: politica, tradición y estética en el orden social moderno (pp. 13-73). Madrid: Alianza.

Beck, U. (2002). La sociedad del riesgo global. Madrid: Siglo XXI.

Cárdenas, A. (2005). Elementos y dinámicas en las relaciones laborales del teletrabajo en Chile. En Gobierno de Chile, Dirección del Trabajo, Departamento de Estudios. Cuaderno de Investigación No 26. Trabajo a domicilio en el siglo XXI: Tres miradas sobre el teletrabajo (pp. 31-62). Santiago: Gobierno de Chile.

Carrasco, C. \& Vega, P. (2011). Una Aproximación a las condiciones de trabajo en la gran minería de altura. Cuaderno de Investigación No 40. Gobierno de Chile. Dirección del Trabajo, Departamento de Estudios. Santiago: Gobierno de Chile.

Correales, M. \& Gómez, A. (2011). Bienestar psicológico de los mineros de la mina "La Playa" del municipio de Amagá. Trabajo de grado para optar al título de psicólogas. Corporación Universitaria Lasallista. Colombia.

Castells, M. (1999). La era de la información. Vol. I: La Sociedad Red. México D.F.: Siglo XXI.

Castells, M. (2001). La galaxia Internet. Reflexiones sobre Internet, empresa y sociedad. Madrid: Areté.

Gareca, M., Verdugo, R., Briones, J. \& Vera, A. (2007). Salud ocupacional y teletrabajo. Ciencia y Trabajo, 9(25), 85-88.

Giddens, A. (1994). Consecuencias de la modernidad. Madrid: Alianza.

Giddens, A. (1995). Modernidad e identidad del Yo: el Yo y la sociedad en la época contemporánea. Barcelona: Península

Giddens, A. (1996). Modernidad y autoidentidad. En A. Giddens, Z. Bauman, N. Luhmann \& U. Beck. Las consecuencias perversas de la modernidad: modernidad, contingencia y riesgo (pp. 33-71). Barcelona: Anthropos.

Giddens, A. (2004). Sociología. Madrid: Alianza.

Gobierno de Chile, Ministerio del Trabajo y Previsión Social (2003). Fija el texto refundido, coordinado y sistematizado del Código del trabajo. Gobierno de Chile, Ministerio del Trabajo y Previsión Social Santiago, Chile.

Godoy, L. \& Mauro, A. (2001). Las relaciones de pareja y los cambios en el mercado de trabajo: el punto de vista de los hombres. Revista de la Academia, 6, 129-147.

González, S., Kreither, J., Lizana, J., Rodríguez, M. \& Zavala, G. (2009). Individuación y modernidad: la constitución de la persona en el espacio público. Revista Austral de Ciencias Sociales, 16, 5-20.

Henríquez, H. (2005). El trabajo a domicilio: una transformación que requiere afinar el diagnóstico. En Gobierno de Chile, Dirección del 
Trabajo, Departamento de Estudios. Cuaderno de Investigación $N^{o}$ 26. Trabajo a domicilio en el siglo XXI: Tres miradas sobre el teletrabajo (pp. 5-30) Santiago: Gobierno de Chile.

Hopenhayn, M. (2004). El Nuevo Mundo del trabajo y los jóvenes. Jóvenes, Revista de Estudios sobre Juventud, 8(20), 54-73.

Jiménez, A. \& Moyano, E. (2008). Factores laborales de equilibrio entre trabajo y familia: Medios para mejorar la calidad de vida. Revista Universum, 23(1), 116-133.

Périlleux, T. (2008). La subjetivación frente a la prueba del trabajo flexible. En A. Soto (editor). Flexibilidad Laboral y Subjetividades: Hacia una comprensión psicosocial del empleo contemporáneo (pp. 137-154). Santiago: LOM.

Selamé, T. (2005). Trabajo a domicilio y teletrabajo: Un estudio de casos en Chile. En Gobierno de Chile, Dirección del Trabajo, Departamento de Estudios. Cuaderno de Investigación No 26. Trabajo a domicilio en el siglo XXI: Tres miradas sobre el teletrabajo (pp. 63-101). Santiago: Gobierno de Chile.

Sennett, R. (2007). La cultura del nuevo capitalismo. Barcelona: Anagrama.

Sisto, V. (2009). Cambios en el trabajo, identidad e inclusión social en Chile: Desafíos para la investigación. Revista Universum, 24(2), 192216.

Soto, A. (2009). Formas y tensiones de los procesos de individualización en el mundo del trabajo. Psicoperspectivas, 7(2), 102-119. Recuperado de http://www.psicoperspectivas.cl

Soto, A. (2011). Narrativas de profesionales chilenos sobre sus trayectorias laborales: la construcción de identidades en el trabajo. Psykhe, 20(1), 15-27.

Soto, A., Espinoza, G. \& Gómez, J. (2008). Aspectos subjetivos vinculados a la flexibilidad laboral. En A. Soto (editor). Flexibilidad Laboral y Subjetividades: Hacia una comprensión psicosocial del empleo contemporáneo (pp. 11-38) Santiago: LOM.

Touraine, A. (1998). Critica a la modernidad. Buenos Aires: Fondo de Cultura Económica.

Fecha de recepción: 16 de enero de 2012.

Fecha de aceptación: 10 de octubre de 2012. 